\title{
Grey extinction in the solar neighbourhood?
}

\author{
W. Skórzyński ${ }^{1}$, A. Strobel ${ }^{1}$, and G. A. Galazutdinov ${ }^{1,2,3}$ \\ 1 Toruń Centre for Astronomy (TCfA), Nicholas Copernicus University, Gagarina 11, 87-100 Toruń, Poland \\ e-mail: red@astri.uni.torun.pl \\ 2 Special Astrophysical Observatory, Nizhnij Arkhyz 369167, Russia \\ e-mail: gala@sao.ru \\ ${ }^{3}$ Isaac Newton Institute of Chile, SAO Branch, Russia
}

Received 21 March 2003 / Accepted 19 June 2003

\begin{abstract}
Some of the close $\mathrm{O}$ and B dwarfs appear to be fainter than indicated by their Hipparcos distances, intrinsic absolute magnitudes attributed to their spectral types, and estimated selective interstellar extinction. This discrepancy is explained in the paper by the grey (neutral) interstellar extinction in the visual range of spectrum. The measure of such an effect is related to discrete features of the interstellar matter.
\end{abstract}

Key words. ISM: dust - extinction

\section{Introduction}

Interstellar dust significantly influences estimates of stellar global characteristics. It changes not only brightness, but also colors of stars. The dependency of the interstellar extinction (comprising both, the absorption and the scatter of light), illustrated by so called Extinction Curve, and discovered by Trumpler (1930a,b), is probably caused by small interstellar dust particles ("Mie" particles).

The absorbing interstellar medium is most likely made of particles of various sizes. The interstellar dust model of $\mathrm{Li}$ \& Greenberg (1997), based on observed extinction curves, counts 3-4 population of grains responsible for the shape of extinction curve at the UV range, but for the extinction at the visual range it proposes only one population - so called large particles of the size about $10^{-8} \mathrm{~m}$. These large particles cause selective extinction at the visual range, but appear to be "grey" at the UV range. For very large particles light obstruction may take place, which will affect all wavelengths alike and cause "neutral" interstellar extinction at both, the visual and UV spectral ranges. We may refer to "grey" or neutral extinction as nonselective extinction.

Since the Trumpler's discovery, it was usually assumed that the total interstellar extinction is proportional to the selective extinction, and that if there is any nonselective component of this extinction, its amount is strictly proportional to the selective as well (Aller \& Trumpler 1939). This has led to the commonly accepted direct proportionality of the total interstellar

Send offprint requests to: A. Strobel,

e-mail: strobel@astri.uni.torun.pl extinction to the measure of its selective effect, the color excess: $A_{v}=R * E(B-V)$, with $R$ being, at least for a given direction in the sky, constant.

Although small particles, responsible for the selective extinction probably predominate in the interstellar space, some regions may offer conditions which allow creation or survival of very large particles as well. The possible presence of such particles was announced and discussed in literature during the last few decades. These were already considered by Trumpler. His early paper (Trumpler 1930b) containes the following statement: "our Milky Way system seems to contain a considerable amount of finely divided matter, noticeable by its absorption of light. This matter appears to be made up mainly of: 1. Free atoms..., 2. Free electrons..., 3. Fine cosmic dust particles of various sizes ... producing the observed selective absorption..., and 4. Perhaps ... also larger meteoric bodies, obstructing light of all wavelengths equally, which may be responsible for a small part of the general absorption (residual effect)". For the residual effect he found an average value of $0.19 \mathrm{mag} / \mathrm{kpc}$, in comparison to the selective one equal $0.31 \mathrm{mag} / \mathrm{kpc}$. Strom et al. (1971), and Strom et al. (1972) have suggested that $\mathrm{A}$ and $\mathrm{F}$ type members of the open cluster NGC 2264, which appear to lie below zero age main sequence (ZAMS) in the $[V,(B-V)]$-plane, are probably surrounded by circumstellar, optically thick disk dust shells having nonselective absorption characteristics at visible wavelengths. Indication of a very high ratio of total to selective absorption for these stars appear to demand the presence of significant number of very large particles in mentioned shells. Jones (1972) similarly explained apparent weakening of some 
members of the Pleiades observed as apparently fainter than their co-members of supposedly the same physical characteristics. Andriesse et al. (1978) in their study on the possible condensation process of solid material around Eta Car, have concluded that such condensate may consist in part of disordered silicate grains with a size about 1 micrometer, which give an almost grey circumstellar extinction of as much as 3-4 mag in the visual and ultraviolet. Dunkin \& Crawford (1998), from localisation of HD 35187B on the HR diagram (obtained with the aid of the reliable Hipparcos distance), have found evidence that this star is dimmed by about 0.4 mag of grey circumstellar extinction. Quite recently Jura et al. (2001) have explained the observed submillimeter fluxes from binaries: 17 Lep, 3 Pup and BM Gem as probably emitted by grains particles as large as $0.1 \mathrm{~mm}$ in radius. They have suggested that circumbinary disc particles may grow by coagulation even to sizes as large as $1 \mathrm{~mm}$. Landgraf et al. (2000) have shown that the grains detected by space probes Ulysses and Galileo extend in their size distibution to 2.0 micrometer, well beyond the maximum sizes commonly adopted for typical interstellar grains, equal 0.25 micrometer, and may be part of the interstellar dust population of the local interstellar cloud. Witt et al. (2001) have examined the grain size distribution that gives rise to the X-ray halo around Nova Cygni 1992 and have suggested to extend the interstellar grain size distribution in the direction to this source to, and possibly even beyond 2.0 micrometer. Such large dust particles are grey at optical and UV wavelengths, and therefore their existence could not be constrained by the extinction curve analysis. Aguirre (1999), and Simonsen \& Hannestad (1999) have demonstrated theoretically that the dimming of distant type Ia supernovae may be caused by large amounts of grey dust in the intergalactic medium. Dust grains can be grey either because they are non-spherical or very large. Moreover, Aguirre (1999) has shown that graphite grains, even as small as $0.1-0.25$ micrometer, would be also too grey to have been detected by its reddening. Finally, as shown Zubko et al. (1996, 1998) such large grains seem to be necessary component of the grain model that match the observed extinction curves at the visual and UV.

It is thus possible, that the total interstellar extinction $\left(A_{v}\right)$ may indeed consists of two components: selective and nonselective (grey $-G E)$.

If $A_{v}$ includes a nonselective component, the contribution of this component may be measured simply by difference between the observed absolute magnitude of a star $M_{v}$ (obs) (derived from its apparent brighteness $(V)$, distance $(d)$, and $A_{v}$ estimated as $R * E(B-V)$ ), and intrinsic absolute magnitude, $M_{v}$ (intr), atributed to spectral type and luminosity class $(\mathrm{Sp} / \mathrm{L})$ of this star by calibration relations:

$G E=M_{v}(\mathrm{obs})-M_{v}($ intr $)$.

In Fig. $1 M_{v}(\mathrm{obs})$ are compared to $M_{v}$ (intr) (taken from calibration relations of Schmidt-Kaler 1982) for a group of close (up to $400 \mathrm{pc}$ ) early type stars. The discrepancies between both mentioned absolute magnitudes are clearly seen in this figure.

Quite formally, a difference (1) may result from a spread of physical properties of stars of the same spectral and luminosity class. Lamers et al. (1997) have determined these differences

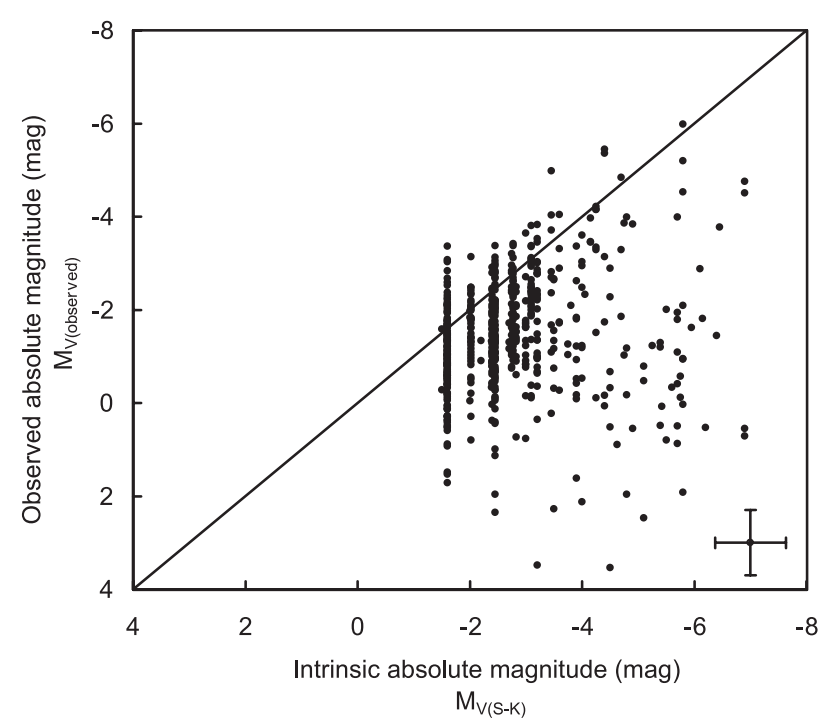

Fig. 1. Comparison of the observed absolute magnitudes with intrinsic ones for all (556) O-B3 stars of different luminosity classes, with distances up to $400 \mathrm{pc}$. The cross represents an average eror bar.

for a few stars and related them to stellar rotation $(v \times \sin i)$. Small sample of stars of different spectral types and luminosity classes used in their analysis create however some doubts about the relation found. Moreover, Jaschek \& Gomez (1998), and Wegner (2000), on the base of much larger samples of early type stars, have not confirmed such a dependency on rotation. Apart of any other stellar physical properties possibly responsible for mentioned difference (like e.g. stellar metallicity), the simplest way to explain such difference seems to relate it to interstellar extinction. Presented in Fig. 1 observed absolute magnitudes have been calculated with the average galactic $R$ value equal 3.1 (Wegner 1993). This gives for the all considered 556 stars the average $G E$ equal 1.3 mag $R$ may vary from place to place in the sky, but rarely, and only occasionally, in some dense molecular clouds, it reach values 4 to 6 (Mathis 1990). If we adopt, for all the stars presented in Fig. 1, $R$ equal 6 , the resulting average $G E$ will still be significant - about 0.84 mag. It is thus very difficult to explain presented $G E$ effect exclusively with unusual $R$ values, and seems reasonable to interpret the $G E$ as possibly related to the additional, neutral component of the interstellar extinction. In the present paper, the $G E$ effect, exhibited by nearby early type stars with reliably determined distances and spectral types is considered in context of its probable relation to the neutral (grey) component of the interstellar dust extinction.

\section{Determination of the GE effect}

In search for a possible non-selective interstellar extinction effects we should concentrate mainly on early type dwarfs. The influence of neutral (grey) extinction will simply "displace" these stars below the zero-age-main-sequence (ZAMS) in the $[V,(B-V)]$-plane (Eq. (1)). For giants or supergiants any misclassification of their luminosity classes pushing them on the $\mathrm{C}-\mathrm{M}$ diagram toward the main sequence, may blur such effects of neutral extinction. 

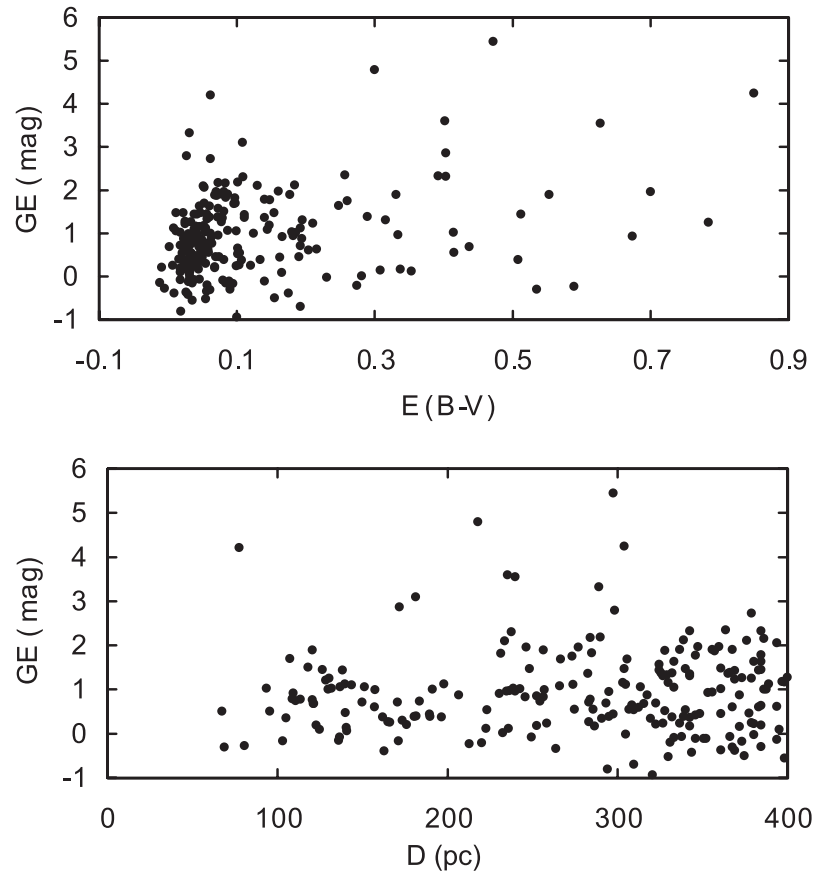

Fig. 2. The derived $G E$ effect vs. $E(B-V)$ and Hipparcos distances.

The reliable $G E$ values may be determined only for stars with precisely determined distances. The Hipparcos - Tycho missions have provided us with a large amount of homogeneous, high quality trigonometric parallaxes and photometric data for many stars. The Hipparcos Catalog (ESA 1997) contains positions, proper motions and trigonometric parallaxes with precisions much better than all previously available for bright $\mathrm{O}$, B stars. These, together with the Tycho photometric data allow to calculate the reliable $G E$ values from:

$G E=V-5 \log (d)+5-3 \cdot 1 E(B-V)-M_{v}($ intr $)$.

For determination of the $G E$ we have used Hipparcos distances, intrinsic absolute magnitudes taken from the $M_{v}$ vs. Sp/L calibrations of Schmidt-Kaler (1982) $\left(M_{v}(\mathrm{~S}-\mathrm{K})\right)$, intrinsic color indices, $(B-V)$ o, from the Papaj et al. (1993), $V$ and $(B-V)$ values from the Hipparcos Catalog, and $R=3.1$ according to Mathis (1990) and Wegner (1993). The spectral types and luminosity classes have been taken from the Hipparcos Catalog. The errors of the differences between observed and intrinsic absolute magnitudes $(G E)$ have been determined according to the Eq. (1) in the paper of Lamers et al. (1997).

To avoid uncertain parallaxes measurements, we have chosen from the Hipparcos Catalog all O-B3 dwarfs with the Hipparcos distances up to $400 \mathrm{pc}$. The resulting sample consists of 220 stars. To check, if the proposed $G E$ is indeed nonselective, $G E$ values, as calculated from (2) for all chosen dwarfs, are drawn against $E(B-V)$ in the upper panel of Fig. 2. No relation between both parameters is seen in this picture. Moreover, even slightly reddened stars show significant $G E$ effect.

The lower panel of Fig. 2 shows that there is no dependency of $G E$ on distance as well. However, as it is shown in Fig. 3, the measure of the selective interstellar extinction, $E(B-V)$, for the same stars, seems also not to depend on distance. This may

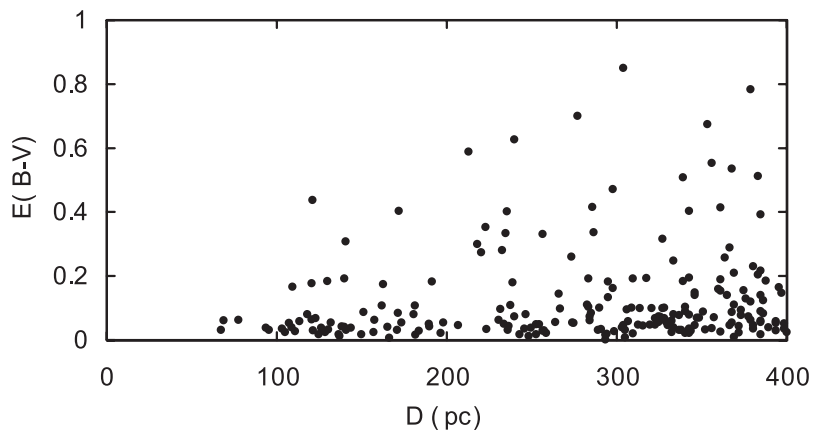

Fig. 3. $E(B-V)$ vs. Hipparcos distances.

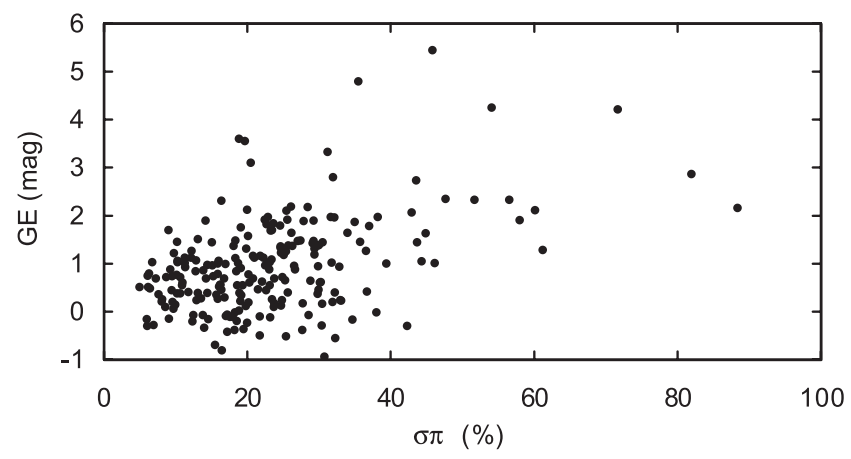

Fig. 4. The dependency of the $G E$ on errors in the Hipparcos trigonometric parallaxes.

indicates for more complex distribution and physical properties of the interstellar dust in the local galactic space.

In Fig. 4 the obtained $G E$ values are drawn against parallaxes errors as cited in the Hipparcos Catalog. As it is seen the $G E$ values seem not depend on parallax errors. Moreover, the picture shows that most of considered stars have parallaxes determined with precision better than 40 percent.

To examine the $G E$ effect more in detail, we have extracted from the above considered sample only stars with trigonometric parallaxes measured with the precision better than 15 percent. In Fig. $5 G E$ values for such $56 \mathrm{O}$ and B dwarfs are drawn vs. $E(B-V)$ (upper panel), and against the Hipparcos distances (lower panel). Again, as in Fig. 2, the $G E$ effect for these more precisely measured stars seems not depend on $E(B-V)$, but seems reach its maximum at distance fro 110 to $150 \mathrm{pc}$.

This suggest, that the considered $G E$ effect may indeed represents non-selective interstellar extinction. Moreover, lack of any distinct dependency of both, $G E$ and $E(B-V)$ on distances may suggests, that the matter responsible for both mentioned components of interstellar extinction is in the solar neighbourhood distributed very unhomogeneously.

\section{Relation of the GE effect to observational features of the interstellar matter}

The derived results presented in last figures motivate to search for possible relations of the $G E$ effect to observational characteristics of the interstellar matter. Unfortunately, general scarcity of data on interstellar matter properties toward particular stars force to include into consideration stars of different 

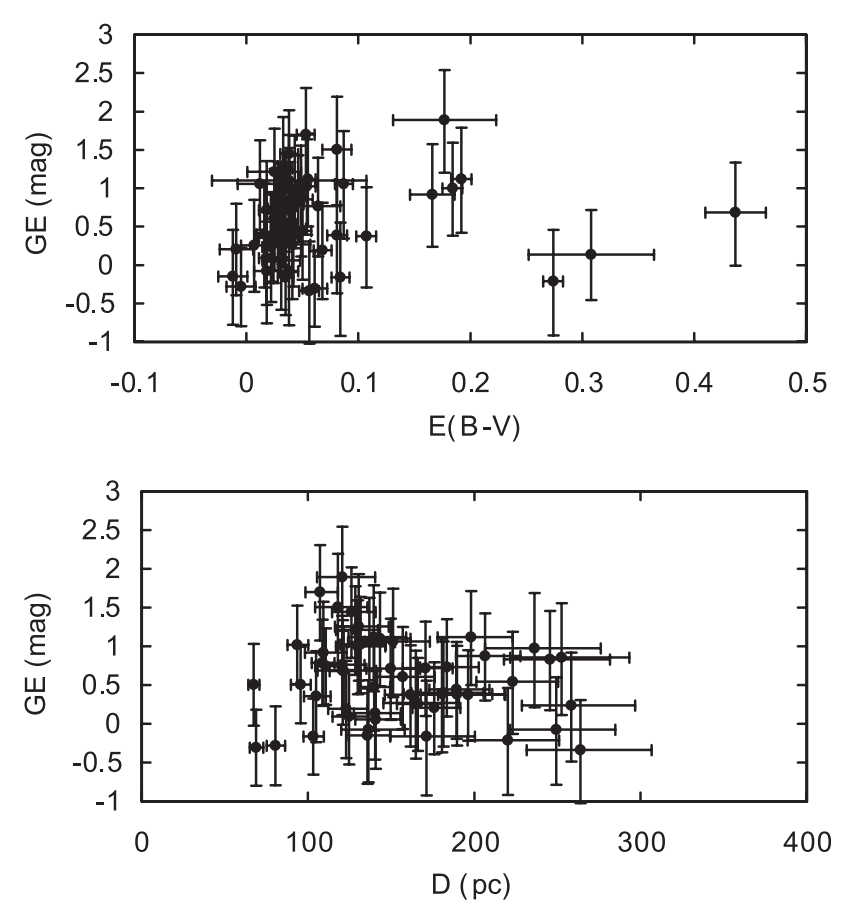

Fig. 5. The $G E$ vs. $E(B-V)$ and Hipparcos distances for stars with the parallaxes errors less than 15 percent.

spectral and luminosity classes, and also stars with worse determined parallaxes. Use of unhomogeneous data may introduce significant scatter in relations studied. This should be taken into account in the interpretation of obtained results.

\subsection{Interstellar krypton}

As a good estimation of the total amount of interstellar matter in particular direction we may use observed abundance of interstellar noble gases, like e.g. argon or krypton. Being chemically neutral, their total abundances along the line of sight are fully revealed by intensities of spectral lines of these elements. Unfortunately, determinations of the abundance of interstellar argon from spectra of $\mathrm{O}$ and $\mathrm{B}$ stars are very rare, and does not allow any statistical considerations. A slightly better situation is with the data for interstellar $\mathrm{Kr} \mathrm{I}$, for which abundances for 15 early type stars were determined by Cartledge et al. (2001) and Cardelli \& Meyer (1997). These Kr I abundances are drawn in Fig. 6, in relation to the total interstellar extinction (selective, estimated as $3.1 E(B-V))+$ non-selective ( $G E$ values $)$ ).

Despite possible large errors, due e.g. to a mix of different spectral types, luminosity classes, or parallax errors of stars presented in the figure, the resulting relation, with correlation coefficient $r=0.89$, seem to suggest a direct relation of the $G E$ to intestellar matter. All the data used in Fig. 6 are contained in the Table 1 .

\subsection{Diffuse interstellar bands}

Among spectral features evidently related to the intestellar matter there are the so called Diffuse Interstellar Bands (DIBs).

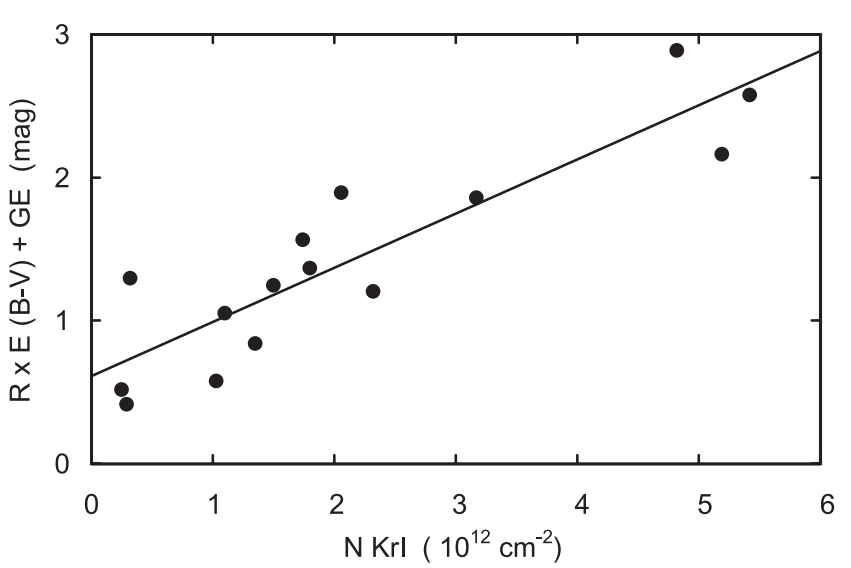

Fig. 6. The relation between column densities of interstellar krypton, and total extinction including $R \times E(B-V)$ and $G E$ values. The average error of krypton column densities is about 15 percent.

Since their discovery by Heger (1922), DIBs were intensively studied, but remain still unidentified. Merrill \& Wilson (1938) proved their strengths to be related to colour excess. These authors were also the first to speculate that DIBs might be "produced by small solid particles". The latest surveys of DIBs have revealed more than 250 such features (Galazutdinov et al. 2000), most however, are very weak. Such weak features can be detectable only in spectra of very high signal to noise ratio $(S / N)$. High quality spectra, necessary to make measurements of such weak structures possible, were obtained with the Sandiford echelle spectrograph fed with the $2.1 \mathrm{~m}$ telescope at McDonald Observatory (Texas USA), and coudé echelle spectrometer fed by the two meter telescope at Terscol Observatory (Northern Caucasus, Russia). The $S / N$ ratios for spectra of individual stars are typically about 500 . The observations have been made by one of us (G.G) at the Terskol Observatory, and by J. Krelowski at the McDonald Observatory.

Inspection of the DIBs revealed some of them being well correlated with the above mentioned $G E$ effect. Average profiles of two such features, centred at about 6367 and $6425 \mathrm{~A}$, are presented in Fig. 7. Profiles of these DIBs for three individual stars of different $G E$ values are shown in Fig. 8. Growth of these DIBs strength with that of $G E$ value is clearly seen in the last figure. From available set of spectra of good quality, we have selected 34 early type stars for which $G E$ values can be determined. Equivalent widths $(W)$ of two mentioned DIBs (6367 and $6425 \AA$ ) are plotted in Figs. 9 and 10 versus the selective $(R E(B-V))$, and grey extinction $(G E)$. These figures show two interesting facts. First, intensity for both DIBs is well correlated with $G E$ effect (correlation coefficients, $r$, are 0.94 for 6367, and 0.93 for 6425). Second, $G E$ is better correlated to the DIB's strength, than to the selective extinction $-R E(B-V)(r=0.68$ for 6367 , and $r=0.67$ for 6425$)$. It should be stressed, that measures compared in these figures are completely independent. The intensity of spectral features $(W)$ have been determined directly from stellar spectra, while $G E$, as well as $R E(B-V)$, were derived exclusively from observed global stellar characteristics. Relations shown in Figs. 9 and 10 strongly support the relation of the measured $G E$ effect to the interstellar matter rather than to the intrinsic global stellar 
Table 1. The gray extinction for stars with known krypton column densities.

\begin{tabular}{|c|c|c|c|c|c|c|c|c|c|c|c|}
\hline HD & Hipp & $\overline{\mathrm{Sp}}$ & $\begin{array}{c}\pi \\
{[\mathrm{mas}]}\end{array}$ & $\begin{array}{c}\sigma \pi \\
{[\mathrm{mas}]}\end{array}$ & $\begin{array}{c}D_{\text {Hipp }} \\
{[\mathrm{pc}]}\end{array}$ & $\begin{array}{c}V \\
{[\mathrm{mag}]}\end{array}$ & $\begin{array}{c}(B-V) \\
{[\mathrm{mag}]}\end{array}$ & $\begin{array}{c}E(B-V) \\
{[\mathrm{mag}]}\end{array}$ & $\begin{array}{c}G E \\
{[\mathrm{mag}]}\end{array}$ & $\begin{array}{c}(\mathrm{KrI}) \\
{\left[10^{12} \mathrm{~cm}^{-2}\right]}\end{array}$ & $\begin{array}{c}\sigma N(\mathrm{KrI}) \\
{\left[10^{12} \mathrm{~cm}^{-2}\right]}\end{array}$ \\
\hline 24398 & 18246 & B1Ib & 3.32 & 0.75 & 301 & 2.84 & 0.27 & 0.46 & $-0.18_{-0.83}^{+0.72}$ & 1.5 & 0.1 \\
\hline 24760 & 18532 & B0,5V & 6.06 & 0.82 & 165 & 2.9 & -0.20 & 0.04 & $0.29_{-0.72}^{+0.68}$ & 0.29 & 0.05 \\
\hline 27778 & 20533 & B3V & 4.49 & 1.11 & 223 & 6.34 & 0.17 & 0.35 & $0.11_{-1.94}^{+0.90}$ & 2.32 & 0.26 \\
\hline 37061 & 26258 & B1V & 2.77 & 0.88 & 361 & 6.75 & 0.18 & 0.41 & $0.88_{-119}^{-1.04}$ & 5.19 & 0.35 \\
\hline 37128 & 26311 & B0Ia & 2.43 & 0.91 & 412 & 1.69 & -0.18 & 0.04 & $0.41_{-1.22}^{+0.19}$ & 0.25 & 0.05 \\
\hline 37903 & 26816 & $\mathrm{~B} 2 \mathrm{~V}$ & 2.12 & 1.23 & 472 & 7.81 & 0.08 & 0.29 & $1.00_{-2.24}^{+1.22}$ & 2.06 & 0.27 \\
\hline 38771 & 27366 & B0,5Ib & 4.52 & 0.77 & 221 & 2.07 & -0.17 & 0.03 & $1.20_{-0.68}^{+0.61}$ & 0.32 & \\
\hline 143275 & 78401 & BOV & 8.12 & 0.88 & 123 & 2.29 & -0.12 & 0.08 & $0.39_{-0.65}^{-0.68}$ & 1.35 & \\
\hline 144470 & 78933 & B1V & 7.7 & 0.87 & 130 & 3.93 & -0.05 & 0.18 & $0.99_{-0.62}^{-0.55}$ & 1.74 & \\
\hline 147888 & 80461 & B3/B4V & 7.33 & 1.37 & 136 & 6.75 & 0.27 & 0.44 & $1.22_{-0.87}^{-0.62}$ & 5.42 & 0.36 \\
\hline 149757 & 81377 & O9,5V & 7.12 & 0.71 & 140 & 2.54 & 0.04 & 0.31 & $0.10_{-0.60}^{+0.58}$ & 1.1 & 0.2 \\
\hline 175360 & 92931 & B6III & 3.66 & 0.85 & 273 & 5.91 & -0.02 & 0.11 & $0.24_{-1.06}^{+1.00}$ & 1.03 & 0.2 \\
\hline 185418 & 96608 & $\mathrm{~B} 0,5 \mathrm{~V}$ & 1.45 & 0.91 & 690 & 7.45 & 0.15 & 0.39 & $0.66_{-254}^{+1.18}$ & 3.17 & 0.46 \\
\hline 203532 & 106474 & $\mathrm{~B} 3 \mathrm{IV} / \mathrm{V}$ & 4.01 & 0.54 & 249 & 6.35 & 0.09 & 0.26 & $0.56_{-0.54}^{+0.54}$ & 1.8 & 0.4 \\
\hline 207198 & 107374 & O9II & 1.62 & 0.48 & 617 & 5.94 & 0.31 & 0.58 & $1.10_{-0.91}^{-0.54}$ & 4.82 & 1.02 \\
\hline
\end{tabular}
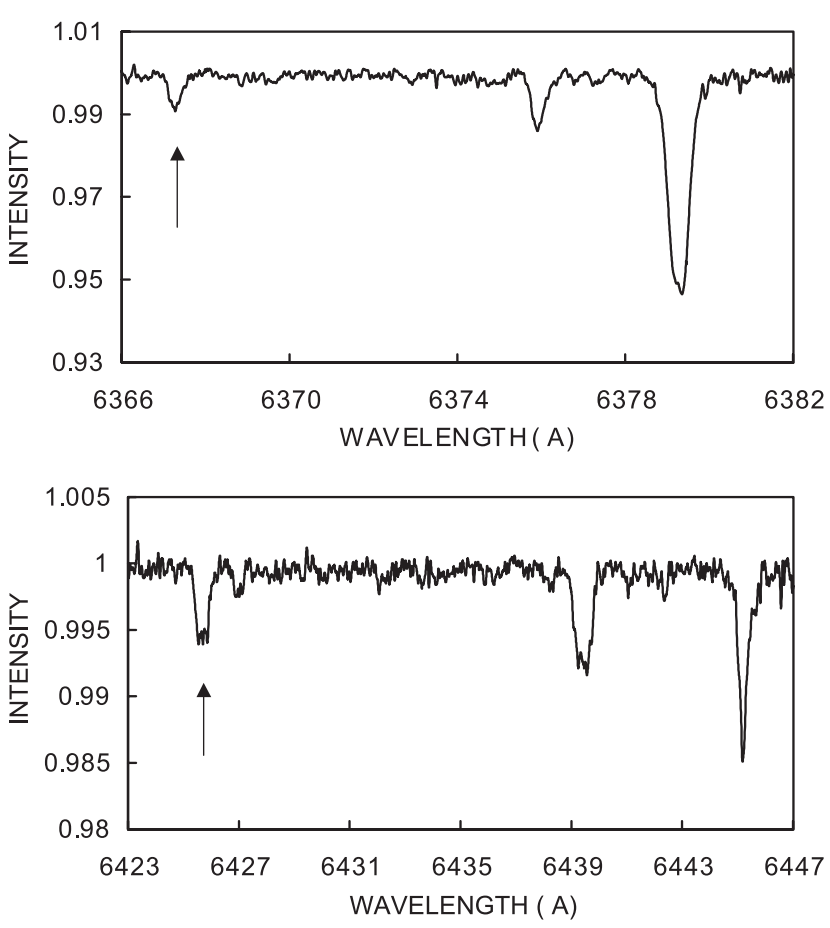

Fig. 7. Spectrum at the vicinity of the two considered DIBs $(6367$ and $6425 \AA$ ), indicated with the arrows. The ordinate scale is the intensity relative to the continuum. To to increase signal-to-noise ratio of these weak DIB's, the both presented fragments are average of 15 and 20 individual spectra, respectively, of three stars (HD 23180, HD 24912 and HD 24398). There are also visible other DIBs (much stronger) centred at 6375.95, 6379.29 $\AA$ (upper panel) and 6439.5, 5445.2 A (lower panel).

characteristics. In Fig. 11 similar relations are presented for the same $34 \mathrm{OB}$ stars for one of the strongest of known DIBs, centred about $5780 \AA$, and in Fig. 12 for another weak DIB, centred at $6194 \AA$. Both these features seem to be better related to the selective $(R E(B-V))$, than to the grey $(G E)$ extinction, quite opposite to DIBs at 6367 and $6425 \AA$. This seems not only to
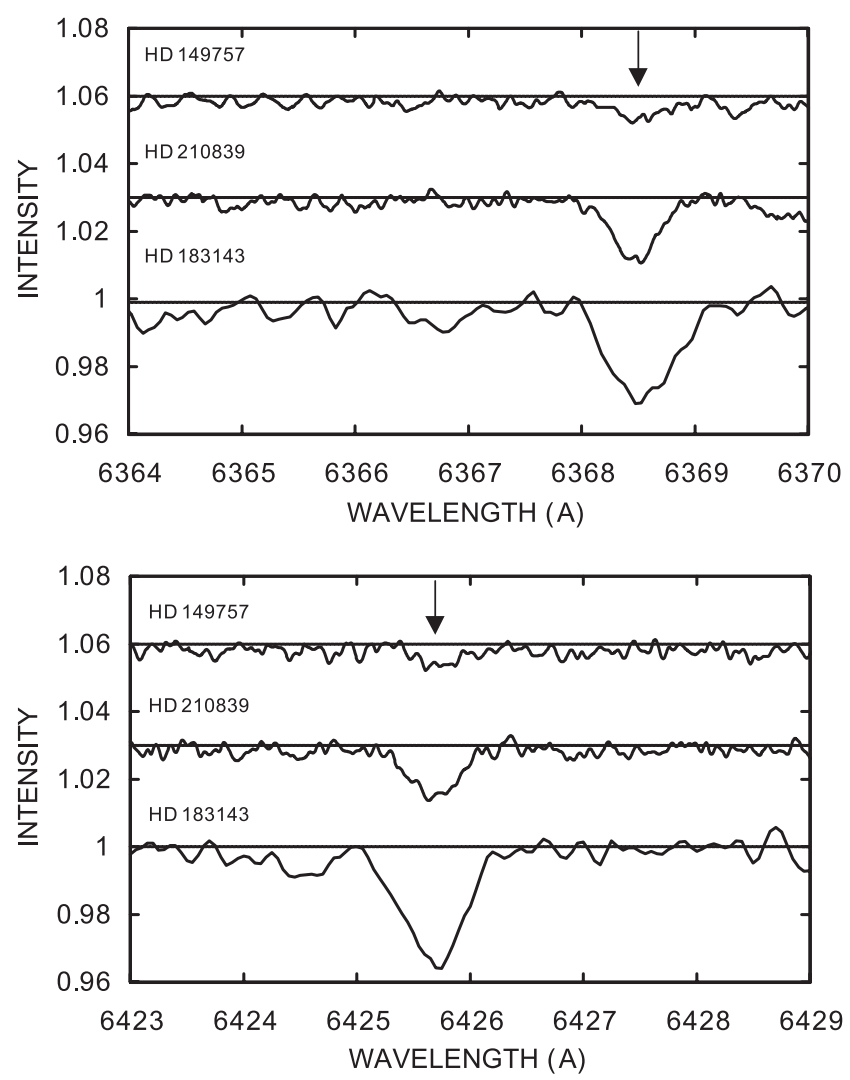

Fig. 8. Comparison of the intensity of the two considered DIBs for three stars with different $G E$ values: HD 149757, $G E=0.10 \mathrm{mag}$, HD 210839, $G E=1.45 \mathrm{mag}$, HD 183143, $G E=2.84 \mathrm{mag}$.

release presented relations from suspicion to be instrumental, or simply artefacts, but suggest that both considered measures $(E(B-V)$ and $G E)$ relate to different interstellar grains populations: the $E(B-V)$ to small size particles, while the $G E$ to very large ones.

The stellar data concernig presented relations of $G E$ to intensities DIB's at 6367 and $6425 \AA$ are listed in the Table 2. 


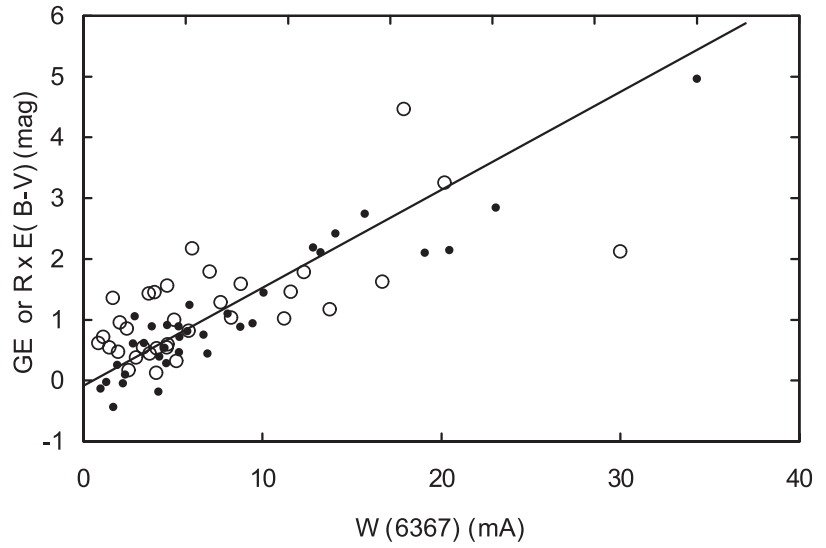

Fig. 9. Equivalent widths of 6367 vs. $G E$ (filled circles- straight line) and vs. $R \times E(B-V)$ (open circles).

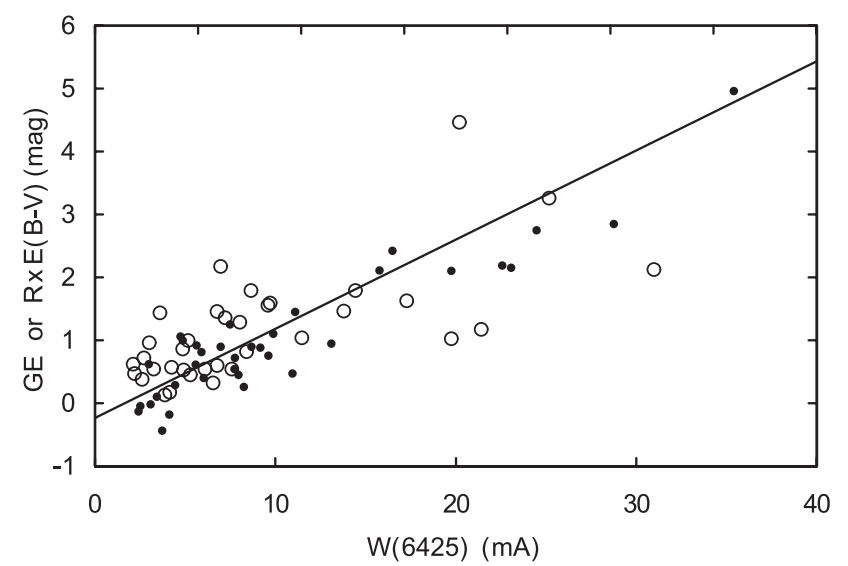

Fig. 10. Equivalent widths of 6425 vs. $G E$ (filled circles- straight line) and vs. $R \times E(B-V)$ (open circles).

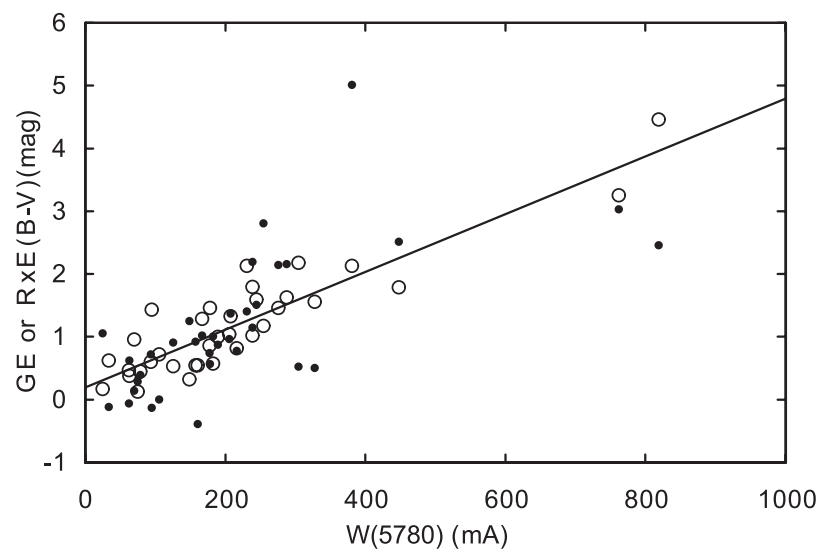

Fig. 11. Equivalent widths of 5780 vs. $G E$ (filled circles) and vs. $R \times E(B-V)$ (open circles- straight line). The data for equivalent widths for 21 stars were taken from Krelowski et al. (1999), for the remaining 13 were determined in the present paper.

\subsection{Polarization}

Interstellar polarization is mainly caused by the interstellar dust grains. The relation of the colour excess, $E(B-V)$ to the

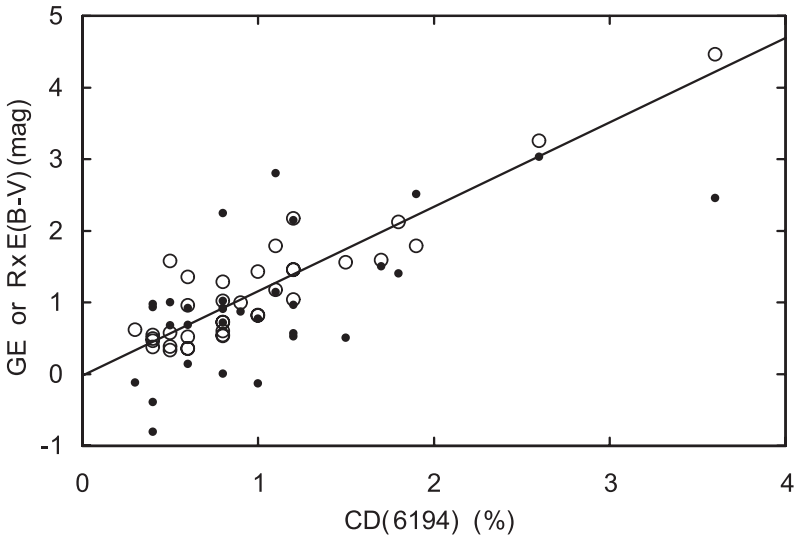

Fig. 12. Central depths (in percentage of the continuum) of DIB centred at $6194 \AA$ vs. $G E$ (filled circles) and vs. $R \times E(B-V)$ (open circles- straight line).
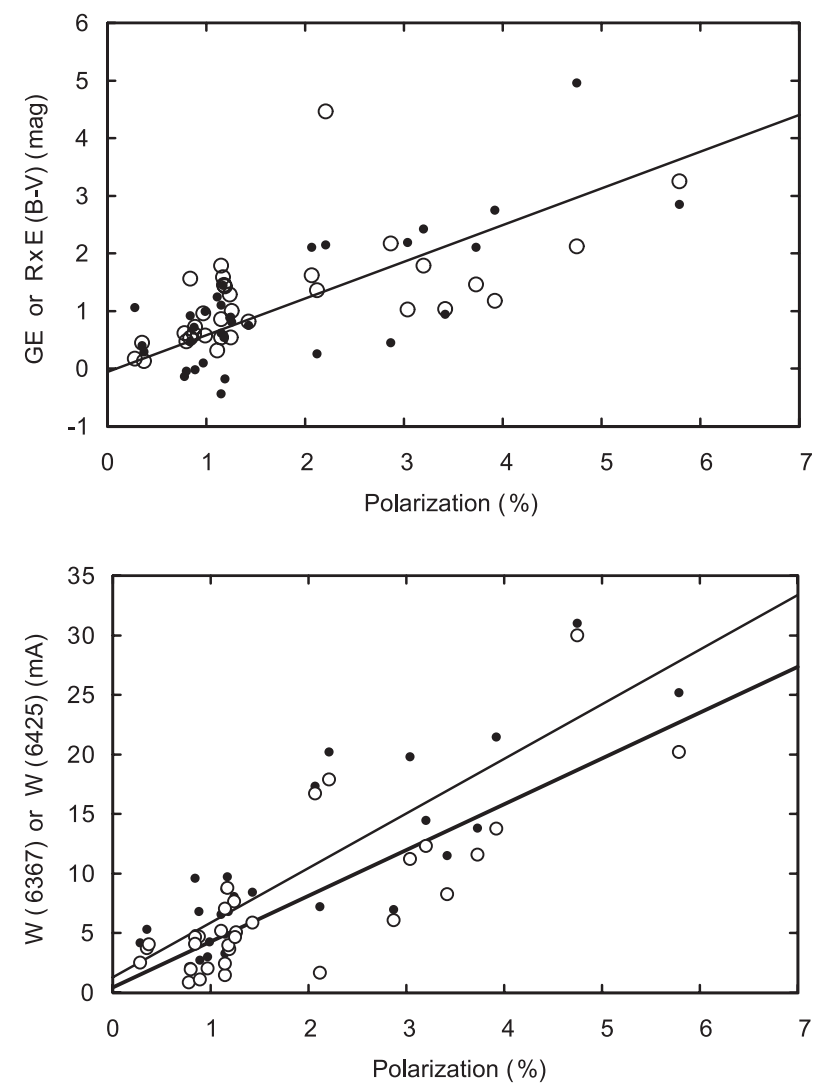

Fig. 13. Upper panel: relation of $G E$ (filled circles- straight line) and $R \times s E(B-V)$ (open circles) to degree of interstellar polarization. Lower panel: relation of the equivalent width of 6367 (open circlesthick line) and 6425 (filled circles-thin line) to interstellar polarization. Typical errors of used polarization data are 10 to 20 percent.

degree of polarization is a well known, and confirm of origin of the selective interstellar extinction from scatter of stellar light on small size dust particles. It is interesting to check, how the measured $G E$ effect depends on polarization. The upper panel of Fig. 13 presents relation of considered measures of supposed two components of the interstellar extinction, selective $(R E(B-V))$, and non-selective $(G E)$, to the polarization degree. The $G E$ effect seems to reveal quite good correlation with 
Table 2. The grey extinction for stars with measured equivalent width of two DIBs 6367 and 6425 .

\begin{tabular}{|c|c|c|c|c|c|c|c|c|c|c|c|}
\hline HD & Hipp & $\mathrm{Sp}$ & $\begin{array}{c}\pi \\
{[\mathrm{mas}]}\end{array}$ & $\begin{array}{c}\sigma \pi \\
{[\mathrm{mas}]}\end{array}$ & $\begin{array}{c}D_{\text {Hipp }} \\
{[\mathrm{pc}]}\end{array}$ & $\begin{array}{c}V \\
{[\mathrm{mag}]}\end{array}$ & $\begin{array}{c}(B-V) \\
{[\mathrm{mag}]}\end{array}$ & $\begin{array}{c}E(B-V) \\
{[\mathrm{mag}]}\end{array}$ & $\begin{array}{c}G E \\
{[\mathrm{mag}]}\end{array}$ & $\begin{array}{c}W 6367 \\
{[\mathrm{~mA}]}\end{array}$ & $\begin{array}{c}W 6425 \\
{[\mathrm{~mA}]}\end{array}$ \\
\hline 5394 & 4427 & B0IV:evar & 5.32 & 0.56 & 188 & 2.15 & -0.05 & 0.20 & $-0.14_{-0.64}^{+0.62}$ & $0.83 \pm 0.16$ & $2.13 \pm 0.40$ \\
\hline 13267 & 10227 & B5Ia & 1.28 & 0.7 & 781 & 6.38 & 0.30 & 0.38 & $2.74_{-2.12}^{+1.35}$ & $13.77 \pm 2.06$ & $21.43 \pm 1.62$ \\
\hline 13854 & 10633 & B1Iab & 1.37 & 0.7 & 730 & 6.48 & 0.28 & 0.47 & $2.10_{-1.83}^{+1.17}$ & $11.59 \pm 1.54$ & $13.82 \pm 1.14$ \\
\hline 15785 & 12009 & B1Iab & 2.9 & 1.05 & 345 & 8.37 & 0.50 & 0.69 & $4.96_{-1.25}^{+0.95}$ & $30.00 \pm 5.00$ & $31.00 \pm 5.00$ \\
\hline 22951 & 17313 & $\mathrm{~B} 0,5 \mathrm{~V}$ & 3.53 & 0.88 & 283 & 4.97 & -0.05 & 0.19 & $0.71_{-1.02}^{+0.88}$ & $4.70 \pm 0.46$ & $6.79 \pm 0.70$ \\
\hline 24398 & 18246 & B1Ib & 3.32 & 0.75 & 301 & 2.84 & 0.27 & 0.46 & $-0.18_{-0.81}^{+0.69}$ & $3.66 \pm 0.31$ & $3.62 \pm 0.27$ \\
\hline 24760 & 18532 & $\mathrm{~B} 0,5 \mathrm{~V}$ & 6.06 & 0.82 & 165 & 2.9 & -0.20 & 0.04 & $0.29_{-0.72}^{+0.68}$ & $4.06 \pm 0.74$ & $3.89 \pm 0.53$ \\
\hline 24912 & 18614 & O7,5Iab: & 1.84 & 0.7 & 543 & 3.98 & 0.02 & 0.32 & $0.81_{-1.16}^{+0.83}$ & $5.08 \pm 0.52$ & $5.18 \pm 0.43$ \\
\hline 34078 & 24575 & O9,5Vvar & 2.24 & 0.74 & 446 & 5.99 & 0.20 & 0.47 & $0.54_{-1.24}^{+0.99}$ & $3.97 \pm 0.52$ & $6.79 \pm 0.52$ \\
\hline 37061 & 26258 & B1V & 2.77 & 0.88 & 361 & 6.75 & 0.18 & 0.41 & $0.88_{-1.19}^{+0.96}$ & $7.66 \pm 0.57$ & $8.03 \pm 0.77$ \\
\hline 40111 & 28237 & $\mathrm{~B} 1 \mathrm{Ib}$ & 1.55 & 0.84 & 645 & 4.81 & -0.09 & 0.10 & $1.25_{-1.95}^{+1.19}$ & $5.19 \pm 0.68$ & $6.57 \pm 0.66$ \\
\hline 43384 & 29840 & B3Ia & 1.56 & 0.87 & 641 & 6.24 & 0.45 & 0.58 & $2.42_{-2.12}^{+1.31}$ & $12.32 \pm 1.39$ & $14.44 \pm 1.42$ \\
\hline 142114 & 77840 & $\mathrm{~B} 2,5 \mathrm{Vn}$ & 7.52 & 1.18 & 133 & 4.59 & -0.07 & 0.12 & $0.62_{-0.77}^{+0.72}$ & $2.95 \pm 0.41$ & $2.62 \pm 0.30$ \\
\hline 143275 & 78401 & B0V & 8.12 & 0.88 & 123 & 2.29 & -0.12 & 0.14 & $0.39_{-0.65}^{+0.62}$ & $3.70 \pm 0.38$ & $5.28 \pm 0.71$ \\
\hline 144217 & 78820 & $\mathrm{~B} 0,5 \mathrm{~V}$ & 6.15 & 1.12 & 163 & 2.56 & -0.07 & 0.18 & $-0.44_{-0.84}^{+0.76}$ & $1.45 \pm 0.14$ & $3.27 \pm 0.38$ \\
\hline 144470 & 78933 & B1V & 7.7 & 0.87 & 130 & 3.93 & -0.05 & 0.18 & $0.99_{-0.62}^{+0.59}$ & & $4.27 \pm 0.32$ \\
\hline 145502 & 79374 & B2IV & 7.47 & 1.11 & 134 & 4.00 & 0.08 & 0.28 & $0.61_{-0.71}^{+0.66}$ & $2.42 \pm 0.28$ & $4.89 \pm 0.49$ \\
\hline 147933 & 80473 & $\mathrm{~B} 2 \mathrm{~V}$ & 8.27 & 1.18 & 121 & 4.57 & 0.23 & 0.44 & $0.25_{-0.69}^{+0.65}$ & $1.65 \pm 0.27$ & $7.22 \pm 0.71$ \\
\hline 149438 & 81266 & B0V & 7.59 & 0.78 & 132 & 2.82 & -0.21 & 0.05 & $1.05_{-0.64}^{+0.61}$ & $2.52 \pm 0.41$ & $4.16 \pm 0.47$ \\
\hline 149757 & 81377 & $09,5 \mathrm{~V}$ & 7.12 & 0.71 & 140 & 2.54 & 0.04 & 0.31 & $0.10_{-0.60}^{+0.58}$ & $2.04 \pm 0.32$ & $3.01 \pm 0.26$ \\
\hline 154445 & 83635 & B1V & 4.26 & 0.96 & 235 & 5.63 & 0.10 & 0.33 & $0.94_{-0.91}^{+0.80}$ & $8.27 \pm 0.49$ & $11.48 \pm 0.58$ \\
\hline 163472 & 87812 & B2IV-V & 3.93 & 0.97 & 254 & 5.82 & 0.06 & 0.26 & $0.75_{-0.87}^{+0.73}$ & $5.87 \pm 0.61$ & $8.42 \pm 0.76$ \\
\hline 168607 & 89956 & B4:Iae & 2.02 & 1.16 & 495 & 8.08 & 1.33 & 1.44 & $2.15_{-2.23}^{+1.36}$ & $17.90 \pm 1.88$ & $20.20 \pm 1.26$ \\
\hline 183143 & 95657 & B7Ia & 2.7 & 0.78 & 370 & 6.84 & 1.01 & 1.05 & $2.84_{-1.19}^{+1.00}$ & $20.18 \pm 1.58$ & $25.18 \pm 0.71$ \\
\hline 184915 & 96483 & B0,5III & 2.24 & 0.81 & 446 & 4.93 & -0.05 & 0.17 & $0.89_{-1.34}^{+1.04}$ & $4.65 \pm 0.61$ & $7.61 \pm 0.65$ \\
\hline 185859 & 96789 & B0,5Ia & 1.15 & 0.78 & 870 & 6.52 & 0.32 & 0.52 & $2.10_{-2.71}^{+1.37}$ & $16.70 \pm 2.00$ & $17.30 \pm 3.00$ \\
\hline 187459 & 97485 & B0,5Ibvar & 1.47 & 0.62 & 680 & 6.42 & 0.13 & 0.33 & $2.19_{-1.41}^{+0.99}$ & $11.22 \pm 2.01$ & $19.77 \pm 3.52$ \\
\hline 198478 & 102724 & B3Ia & 1.45 & 0.55 & 690 & 4.81 & 0.57 & 0.70 & $0.44_{-1.39}^{+1.05}$ & $6.07 \pm 0.38$ & $6.98 \pm 0.60$ \\
\hline 207198 & 107374 & O9II & 1.62 & 0.48 & 617 & 5.94 & 0.31 & 0.58 & $1.10_{-0.91}^{+0.71}$ & $7.05 \pm 0.55$ & $8.66 \pm 0.87$ \\
\hline 210839 & 109556 & O6Iab & 1.98 & 0.46 & 505 & 5.05 & 0.19 & 0.51 & $1.45_{-0.72}^{+0.60}$ & $8.80 \pm 0.91$ & $9.73 \pm 0.61$ \\
\hline 213087 & 110817 & B0,5Ib & 1.29 & 0.55 & 775 & 5.52 & 0.30 & 0.50 & $0.47_{-1.43}^{+1.00}$ & $4.67 \pm 0.50$ & $9.59 \pm 1.95$ \\
\hline 216200 & 112778 & B3IV:var & 3 & 0.75 & 333 & 5.91 & 0.06 & 0.23 & $-0.02_{-1.01}^{+0.87}$ & $1.11 \pm 0.25$ & $2.71 \pm 0.25$ \\
\hline 218376 & 114104 & B0,5IV & 2.95 & 0.53 & 339 & 4.84 & -0.06 & 0.17 & $0.91_{-0.80}^{+0.73}$ & $4.10 \pm 0.42$ & $4.93 \pm 0.34$ \\
\hline 223128 & 117265 & B2IV & 1.88 & 0.6 & 532 & 5.95 & -0.05 & 0.15 & $-0.05_{-1.19}^{+0.96}$ & $1.94 \pm 0.29$ & $2.20 \pm 0.32$ \\
\hline
\end{tabular}

the polarization ( $r=0.77$ ), not worse than that of selective extinction $(r=0.59)$. Similarly good correlation with interstellar polarization is exhibited also by the intensities of both above mentioned DIB's: at 6367 and $6425 \AA$ as is shown in the lower panel of Fig. 13 ( $r=0.80$ and $r=0.85$, respectively).

All the data on polarization have been taken from Mathewson et al. (1978). Presented correlations of $G E$ and DIB's intensities with degree of interstellar polarization seem to suggest their direct relation to interstellar dust grains.

\section{Conclusions}

The performed analysis of the available astrometric, spectroscopic and photometric data for early type stars in the solar neighborhood indicate, that observed weakening brighteness of many of these in comparison to expected from their Hipparcos distances and intrinsic absolute magnitudes, called by us the $G E$ effect, may be explained by the existence along observed directions, of large interstellar grains causing neutral (grey) extinction, similar to those considered in the literature.

The interstellar extinction interpretation of the $G E$ effect seems to be supported by distinct correlation between the adopted $G E$ measure (Eqs. (1) and (2)), and the total amount of the interstellar matter indicated by the column density of the noble gas (Kr I) or intensities of some diffuse interstellar bands (e.g. those centred at $6367 \AA$ and $6425 \AA$ ).

Surprisingly good correlation between $G E$ and $W$ of the two mentioned DIBs over large range of $G E$, as presented in 

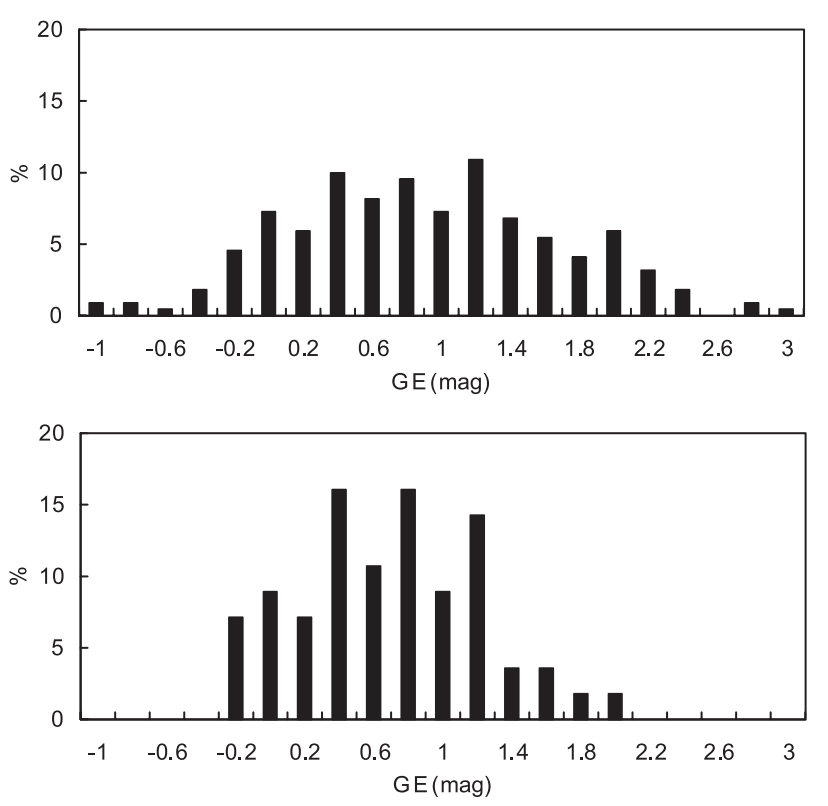

Fig. 14. The percentage distribution of the $G E$ effect for 220 O-B3 dwarfs up to $400 \mathrm{pc}$ (upper panel) and subsample of the same stars with parallax errors less then 15 percent (lower panel).

Figs. 9 and 10, and distinct relation of the $G E$ to the degree of interstellar polarization shown in Fig. 13, seem to strongly suggest, that both, the adopted $G E$ measure, and the two considered DIBs (6367 and $6425 \AA$ ) are related to "super large" interstellar dust particles responsible for the grey (non-selective) interstellar extinction. To illustrate, how significant is this grey (neutral) extinction in the solar neighbourhood, in Fig. 14 are presented histograms of the percentage distribution of the $G E$ values for the both chosen samples of close O-B3 dwarfs (Sect. 2). The upper panel presents the $G E$ distribution for all dwarfs up to $400 \mathrm{pc}$, the lower one for stars with parallaxes errors less than 15 percent. The "zero" on the abscissa indicates the zero difference between absolute magnitudes taken from the Schmidt-Kaler calibration, and those derived from the Hipparcos parallaxes corrected to the selective extinction effects.

The distinct asymmetry in both presented distributions suggests a significant value (up to a few magnitudes!) of possible neutral extinction at the distances up to only $400 \mathrm{pc}$. Such a strong, local neutral extinction can significantly change not only estimation of global physical characteristics of close stars, but also a view of the spatial distribution of objects at the local galactic region.

\section{References}

Aguirre, A. 1999, ApJ, 525, 583

Andriesse, C. D., Donn, B. D., \& Viotti, R. 1978, MNRAS, 185, 771

Aller, L. H., \& Trumpler, R. J. 1939, PASP, 51, 339

Cardelli, J. A., \& Meyer, D. M. 1997, ApJ, 477, 57

Cartledge, S. J. B., Meyer, D. M., Lauroesch, J. T., \& Sofia, U. J. 2001, ApJ, 562, 394

Dunkin, S. K., \& Crawford, I. A. 1998, MNRAS, 298, 275

ESA 1997, The Hipparcos and Tycho Catalogues, ESA SP-1200

Galazutdinov, G. A., Musaev, F. A., Krelowski, J., \& Walker, G. A. H. 2000, PASP, 112, 648

Heger, M. L. 1922, Lick. Obs. Bull. 337, 141

Jaschek, C., \& Gomez, A. E. 1998, A\&A, 330, 619

Jones, B. F. 1972, ApJ, 171, 57

Krelowski, J., Ehrenfreund, O., Foing, B. H., et al. 1999, A\&A, 347, 235

Lamers, H. J. G. L. M., Hazevoort, J. M. A. G., Schrijver, H., et al. 1997, A\&A, 325, 25

Landgraf, M., Baggaley, W. J., Grun, E., Kruger, H., \& Linkert, G. 2000, J. Geophys. Res., 105, 10343

Li, A., \& Greenberg, J. M. 1997, A\&A, 323, 566

Mathewson, D. S., Ford, V. I., Klare, G., Neckel, T., \& Krautter, J. 1978, Bull. Inf. C.D.S., 14, 115

Mathis, J. 1990, An. Rev. Astr. Ap., 28, 37

Merrill, P. W., \& Wilson, O. C. 1938, ApJ, 87, 9

Papaj, J., Krelowski, J., \& Wegner, W. 1993, A\&A, 273, 575

Schmidt-Kaler, Th. 1982, in Landolt-Boernstein, group VI, vol. 2, subvol. b, Stars and Star Clusters, ed. K. Schaifers, \& H. H. Voigt (Berlin, Heidelberg, New York: Springer), 17

Simonsen, J. T., \& Hannestad, S. 1999, A\&A, 351, 1

Strom, K. M., Strom, S. E., \& Yost, J. 1971, ApJ, 165, 479

Strom, S. E., Strom, K. M., Brooke, A. L., Bregman, J., \& Yost, J. 1972, ApJ, 171, 267

Trumpler, R. J. 1930a, Lick Obs. Bull. 14, 154

Trumpler, R. J. 1930b, PASP, 42, 214

Wegner, W. 1993, Act. Astr., 43, 209

Wegner, W. 1994, MNRAS, 270, 229

Wegner, W. 2000, MNRAS, 319, 771

Witt, A. N., Smith, R. K., \& Dwek, E. 2001, ApJ, 550, L201

Zubko, V. G., Krelowski, J., \& Wegner, W. 1996, MNRAS, 283, 577

Zubko, V. G., Krelowski, J., \& Wegner, W. 1998, MNRAS, 294, 548 